\title{
Self-loosening of 3D printed bolted joints for engineering applications
}

\author{
Jun-Hee Wi, Kwang-Hee Lee, and Chul-Hee Lee ${ }^{*}$ \\ Department Mechanical Engineering, Inha University, 100 inha-ro, Nam-gu, Incheon, 22212, South \\ Korea
}

\begin{abstract}
A bolt joint is a simple element that joins mechanical components. Self-loosening of bolted joints occurs due to the vibrations caused by motors and engines, resulting in the breakage of machines, and potentially serious safety problems. Recently, developments in 3D printing technologies have enabled the fabrication of detailed components. These technologies can be used for producing fasteners using $3 \mathrm{D}$ printed bolts. Many researchers have proposed a theoretical model for self-loosening of the bolt, and experimental studies on the self-loosening phenomenon have been advanced. However, studies on the self-loosening of 3D printed bolts have not been conducted. Therefore, it aims to confirm the self-loosening phenomenon and the safety of $3 \mathrm{D}$ printed bolts through experiments and finite element simulation. A lateral vibration test system is constructed and self-loosening of the bolt is evaluated by observing the axial force according to the vibration cycle by using a strain gauge. This study compared the self-loosening of 3D printed bolts and steel bolts by changing the preload and amplitude. In addition, the experimental results are verified through finite element simulation. Through this study, it is expected that 3D printed bolts will be used more frequently in situations where specially shaped bolts are needed.
\end{abstract}

\section{Introduction}

Bolts are the most efficient and simplest parts used to combine mechanical components. The most serious problem associated with the tightening of bolts is self-loosening. Experimental observations indicate that the self-loosening of a bolt begins with the relaxation of the initial preload. The self-loosening in such a scenario is called the first stage of self-loosening of the bolt. After the clamping force drops below a certain value, the nut begins to unwind [1]. This is the second stage of self-loosening of the bolt. If the bolt loosens, it causes a serious safety problem. Many researchers have studied the relevant principles and performed experiments to predict the circumstances under which the bolt self-loosens. Junker suggests that the self-loosening of the bolt has a greater effect when a vibration occurs in the shear direction of the bolt than when a vertical vibration occurs [2]. Therefore, a lateral load test based on the Junker tester has been carried out.

*Corresponding author: chulhee@inha.ac.kr 
Recently, a number of small and complicated parts are being produced by 3D printing methods using various materials because of the advantages of employing 3D technologies in manufacturing. This improvement in 3D printing technologies will enable the use of $3 \mathrm{D}$ printed bolts and nuts as fasteners. However, no research has been conducted on the selfloosening and safety of 3D printed bolts and nuts. Therefore, the self-loosening characteristic of the 3D printed bolt is evaluated in this study and the results are compared with that of the steel bolt. The simulation and experiment are conducted to predict the selfloosening characteristics of steel and 3D printed bolts under various preload and relative displacements of jointed part conditions. The first stage of self-loosening of the bolt not only leads to the second stage of self-loosening but can also lead to a serious accident due to a decrease in clamping force. Therefore, the first stage of self-loosening is only focused in this study. It is assumed that the results obtained from this study can be used as a reference to design structures that use $3 \mathrm{D}$ printed fasteners.

\section{Experiments}

\subsection{D printed specimen}

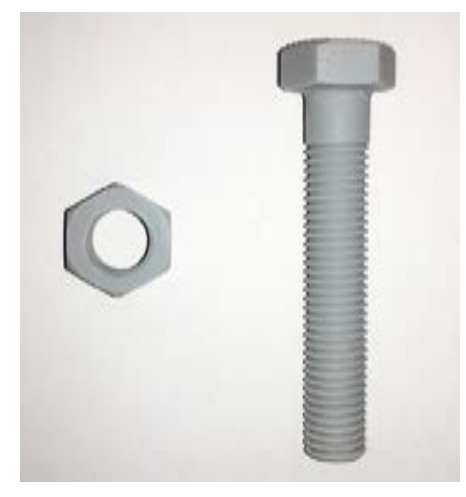

Fig. 1. Specimen of 3D printed bolt and nut.

3D printing technologies have been widely used in various applications where complicated parts are required, because the technologies have the ability to produce parts without additional processes, compared to other manufacturing technologies. The specimen of bolt and nut are 3D printed using Stereolithography Apparatus (SLA) printer (XFAB) as shown in Fig. 1 to evaluate self-loosening characteristic under various operating conditions. The bolts and nuts were printed in the same size as the M12X70 steel bolt.

A tensile test, according to ISO 527, for SLA material (PRECISA 779) was conducted to obtain the material properties of the $3 \mathrm{D}$ printed bolt and nut. The tensile tests were performed with the universal testing machine (UTM). The tensile test resulted on a yield strength of $26 \mathrm{MPa}$. The obtained material properties are used to calculate the appropriate preload of the $3 \mathrm{D}$ printed bolt during the self-loosening tests and the finite element simulations.

\subsection{Test setup for self-loosening of bolted joint}

Experimental equipment is specifically designed to evaluate the self-loosening of bolted joints as shown in Fig. 2. A holder with an eccentricity of $0.25 \mathrm{~mm}, 0.5 \mathrm{~mm}$, and $0.75 \mathrm{~mm}$ 
is connected to the motor (1,500 W, BLDC220 V, 2,000 RPM) shaft and the linear motion (LM) guide. Therefore, the horizontal movement (perpendicular to the axis of bolt) can be provided as a transverse load to the clamped parts. A load cell (BONGSHIN, DBBP-2 $t$ ) is placed be-tween the LM guide and the upper plate of the clamped parts to directly measure the force exerted on the bolt. Several pins (1 mm diameter) are inserted between the two plates so that the relative movement of the plate is more likely to occur. A strain gauge is also attached to the shaft of the bolt to monitor the change in the clamping force in real time. The size of the steel bolt is M12 and its strength grade is 4.6. A rotary type encoder is attached to the end of the bolt and the nut is fixed to the bolt using an adhesive (Epons AR16) to purely monitor the rotation of the bolt in the first stage of self-loosening.

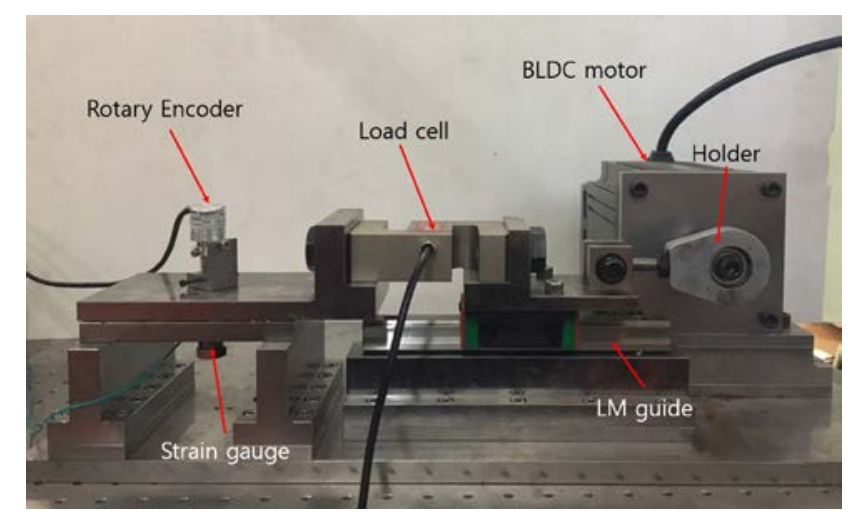

Fig. 2. Experimental equipment for self-loosening of bolted joints under vibration condition.

The test is carried out to observe the self-loosening of the bolt by providing a relative movement between the lower and the upper plates with a constant operating frequency. According to Bickford and Eccles, the appropriate level of preload corresponds to an axial stress is $50 \%$ to $75 \%$ of the nominal yield strength of the bolt $[1,6]$. Based on this, the steel bolt and $3 \mathrm{D}$ printed bolt were tightened with preloads ranging from $9.5 \mathrm{kN}$ and from 1.01 $\mathrm{kN}$, respectively.

The relative displacement condition is altered to monitor how it affects the selfloosening characteristic for the steel bolt specimen and the 3D printed bolt.

\section{Finite element simulations}

A simulation model of bolted joint was configured to evaluate the first stage of selfloosening under vibration conditions. It consists of a bolt, a nut, and two plates. The bolt is modelled using the dimension of M12X70 hex bolt. The 3D finite element model is prepared using Hypermesh as shown in Fig. 3 [7]. This study focuses on observing the first 


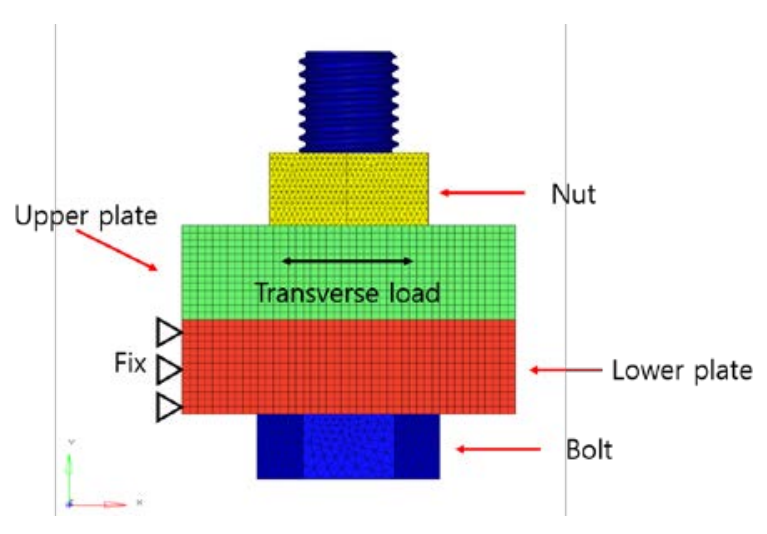

Fig. 3. 3D finite element mesh model.

stage of self-loosening for 3D printed bolt. Therefore, elastic deformations are only considered for the materials used in the simulation. The elastic modulus of the $3 \mathrm{D}$ printing material is $3.9 \mathrm{GPa}$ and Poisson's ratio is 0.3 .

Another important parameter for the analysis is the coefficient of friction between the contact surfaces. The threading friction between the bolt and the nut has been experimentally determined in the previous study and is 0.09 [8]. The coefficient of friction between the upper plate and the lower plate is defined as 0.16 . For other contact interfaces (lower plate-bolt, upper plate-nut), the coefficient of friction is defined as 0.1 .

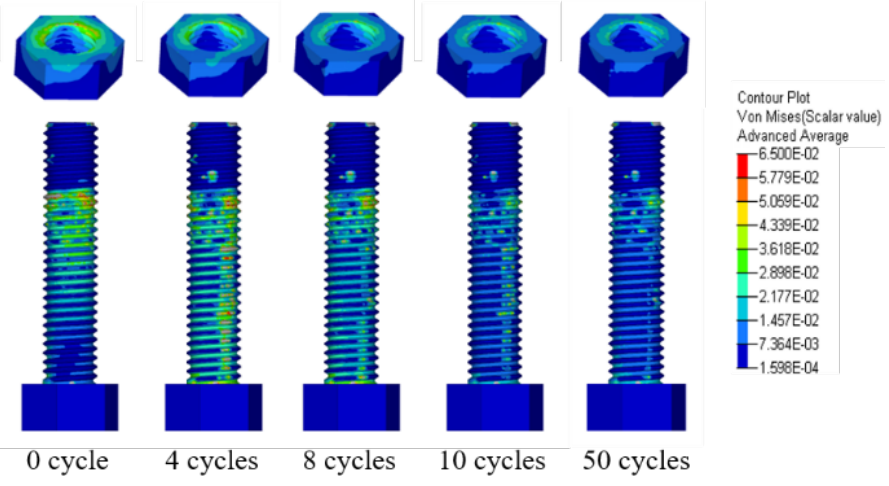

Fig. 4. Stress changes in the 3D printed bolt and nut model during the cycles of transverse load.

$$
\text { (unit GPA) }
$$

The calculated preload is defined by applying the rotating motion of the nut in the jointed parts. The tightening torque is applied by the rotated nut and the required preload is obtained. After that, the simulation of the self-loosening phenomenon is conducted while the relative motion between the fixed lower plate and the upper plate is being applied. The maximum relative displacement is set to $0.25 \mathrm{~mm}$. Each loading case proceeded in 50 cycles. Fig. 4 shows the stress change of the 3D printed bolt during the simulation. During the self-loosening, the stress is observed on the contact surface between the bolt and plates. The stress is reduced when the first stage of self-loosening is finished (after 10 cycles). Therefore, it is assumed that the clamping force is reduced to lower stress in the $3 \mathrm{D}$ printed bolt. 


\section{Results and discussions}

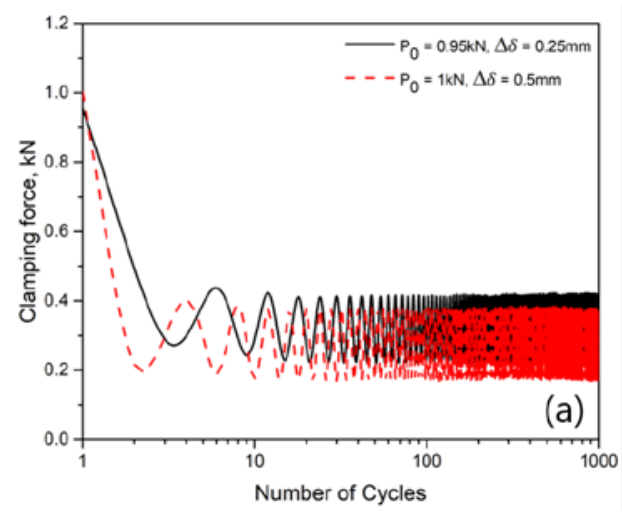

(a) 3D printed bolts

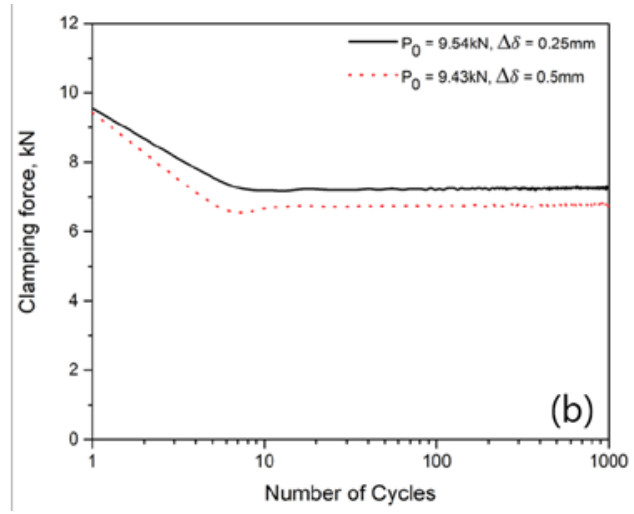

(b) Steel bolts

Fig. 5. Reduction in clamping force of bolts according to the number of cycles.

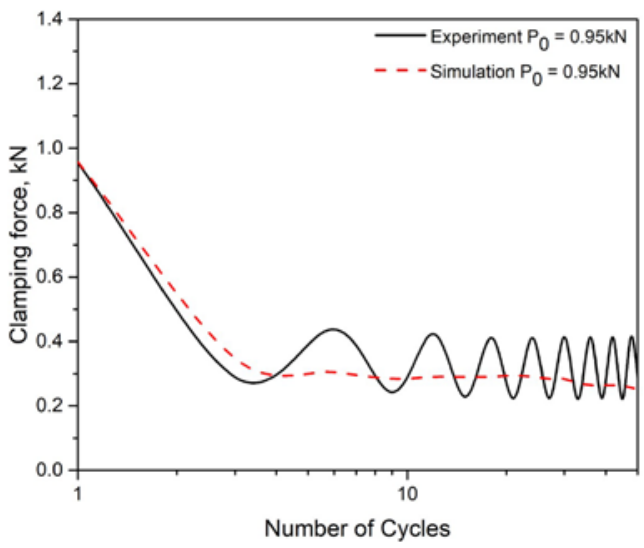

Fig. 6. Comparison of clamping force reduction in simulation and experiment.

Fig. 5 shows the experimental result for the reduction in the clamping force of the 3D printed bolt and steel bolt according to the number of cycles with changes in initial preload and relative displacement. The initial values of preload are set to the calculated values as close as possible and are $50 \%$ of the nominal yield strength. In the Fig. 5(a), the initial preload of the 3D printed bolt is $1.01 \mathrm{kN}$. In Fig. 5(b), the loss of the clamping force is observed when the relative displacements are $0.25 \mathrm{~mm}$ and $0.5 \mathrm{~mm}$ while keeping the initial preload set to $9.5 \mathrm{kN}$. It is assumed that the first stage of self-loosening ends when the clamping force becomes stable. As the amplitude of the relative displacement increases, the slope of the loss of clamping force becomes larger and the first stage of self-loosening occurs earlier. In general, the clamping force slowly decreases after the first stage of selfloosening. However, the clamping force remains constant after the first stage because the nut is fixed to the bolt, preventing the rotating motion of the nut. The self-loosening of the 3D printed bolt begins earlier than that of the steel bolt. 
A graph comparing the self-loosening phenomena of 3D printed bolts with the selfloosening phenomena using finite element (FE) simulation and experiment is shown in Fig. 6 . The preloads for the $3 \mathrm{D}$ printed bolt are set at $0.95 \mathrm{kN}$. The relative displacement of the bolted plates is $0.25 \mathrm{~mm}$. In the FE simulation results, the clamping force decreases and the self-loosening tendency is in good agreement with the experimental results. The same preload for steel bolt cannot be applied to the 3D printed bolt owing to the lower strength of $3 \mathrm{D}$ printing material. However, when specially designed bolts are to be used, they will be more advantageous than steel bolts.

Whether the first stage or second stage has a significant influence on self-loosening process depends on the material of the bolt. For low-class bolts, the first stage of selfloosening becomes dominant. More than $30 \%$ of the clamping force can be reduced in the first stage of self-loosening [9].

In this study, only the first stage of self-loosening, where the rotation of the nut does not occur, is observed. In most previous studies, the second stage of self-loosening of the bolt is considered to be the cause of the self-loosening, and the second stage of self-loosening is considered to be more dangerous than the first stage of self-loosening. However, since the first stage of self-loosening of the bolt leads to the second stage of self-loosening and can cause big accidents, the first stage of self-loosening should also be studied carefully.

\section{Conclusions}

1. The first stage of self-loosening is affected by the material of the bolt as well as the relative displacement of the plate. $3 \mathrm{D}$ printed bolts exhibit a clamping force loss of more than $30 \%$ due to the nature of the material.

2. The FE simulation can be used to identify the first step of the bolt self-loosening. This can be used to remove elements that can cause the second stage of self-loosening before a critical issue occurs.

3. The 3D printed bolt loosens before the steel bolt, but it is more advantageous than the steel bolt in the fastening situation where the special shape is more important. Using materials similar to steel rather than conventional 3D printing materials would be more advantageous than steel bolts in terms of safety and specific design.

\section{References}

1. J.H. Bickford, An Introduction to the Design and Behavior of Bolted Joint (Marcel Dekker, New York, 1995)

2. G.H. Junker, SAE International, SAE Paper 690055 314-335 (1969)

3. R.F. Finkelston, Machine Design, 44 122-125 (1972)

4. T. Sakai, Bulletin of JSME, 21 333-340 (1978)

5. T. Sakai, bulletin of JSME, 21 1385-1390 (1978)

6. W. Eccles, SAE International, SAE Paper 930578 (1993)

7. Hypermesh, On-line Help and Technical Support, Altair Engineering Inc.

8. Y. Jiang, M. Zhang, T.W. Park, C.H. Lee, ASME Pressure Vessel and Piping Conference, 1083 (2002)

9. Y. Jiang, M. Zhang, T.W. Park, C.H. Lee, ASME Journal of Mechanical Design, 125 $518-526(2003)$ 